Mens

revue d'histoire intellectuelle de l'Amérique française

\title{
La rage de Vivre et les « Cahiers noirs » (1934-1935)
}

\section{Yvan Lamonde}

Volume 9, numéro 2, printemps 2009

URI : https://id.erudit.org/iderudit/1023094ar

DOI : https://doi.org/10.7202/1023094ar

Aller au sommaire du numéro

Éditeur(s)

Centre de recherche en civilisation canadienne-française

ISSN

1492-8647 (imprimé)

1927-9299 (numérique)

Découvrir la revue

Citer cet article

Lamonde, Y. (2009). La rage de Vivre et les «Cahiers noirs " (1934-1935). Mens, 9(2), 187-206. https://doi.org/10.7202/1023094ar

\section{Résumé de l'article}

Areligieuse mais politique comme les Jeune-Canada qu'elle considère par ailleurs « bourgeois », la revue Vivre se démarque de la Jeunesse ouvrière catholique, de L'Action nationale et des « métaphysiciens » de La Relève. Éclectique dans ses inspirations politiques et intellectuelles, anticonformiste par son ton, la revue de Québec se met aussi à l'enseigne de Jean-Charles Harvey, dénonciateur des " demi-civilisés ». " Vivre » est pour l'éphémère revue « combattre la peur » et plaider pour la vie, pour le vivant, pour le présent contre le conformisme des peureux qui prennent le passé pour maître. Vivre renaîtra dans La Nation. 


\title{
Articles
}

\section{LA RAGE DE VIVRE \\ ET LES « CAHIERS NOIRS » \\ (1934-1935)}

\author{
Yvan Lamonde \\ Département de langue et de littérature françaises \\ Université McGill
}

\section{Résumé}

Areligieuse mais politique comme les Jeune-Canada qu'elle considère par ailleurs "bourgeois", la revue Vivre se démarque de la Jeunesse ouvrière catholique, de L'Action nationale et des « métaphysiciens » de La Releve. Éclectique dans ses inspirations politiques et intellectuelles, anticonformiste par son ton, la revue de Québec se met aussi à l'enseigne de Jean-Charles Harvey, dénonciateur des « demi-civilisés ». « Vivre » est pour l'éphémère revue « combattre la peur » et plaider pour la vie, pour le vivant, pour le présent contre le conformisme des peureux qui prennent le passé pour maittre. Vivre renaîtra dans La Nation.

\section{Abstract}

The areligious journal Vivre differed significantly from the Jeunesse ouvrière catbolique, L'Action nationale, and the "metaphysicists" of La Relève. However, like the Jeune-Canada movement, which it considered "bourgeois," the Quebec-city based Vivre was quite political. Eclectic in itspolitical and intellectual inspirations and anti-conformist in tone, the journal found one of its leading figure in Jean-Charles Harvey, who is perbaps best known as the denunciator of the "demi-civilisés." For Vivre, "to live" was to "combat fear" and exalt life 
and all that was living. The ephemeral journal, which would eventually reemerge as La Nation, embraced the present and rejected the conformism of anxious intellectuals who looked to the past for inspiration.

«[F]ondée sur un coin de table d'une taverne de la rue Saint-Jean ", à Québec, Vivre paraîtra un an, du 15 mai 1934 au 15 mai 1935, d'abord sous forme de «cahiers » mensuels ronéotypés, puis d'imprimé bimensuel à compter de mars 1935․ Vivre sera relayée par Les Cabiers noirs (1935), puis par La Nation (15 février 1936 - 8 juillet 1939), à telle enseigne que la ville de Québec retrouvera dans la décennie de la Crise une vitalité intellectuelle dont Arthur Buies avait commencé à décrire le déclin vers 1875 . Sur une période de six ans, cette suite de périodiques de la ville de Québec permet de trouver une variante politique du corporatisme et du fascisme, version canadienne-française.

La décision des rédacteurs de bien faire porter son nom à la revue lui donne un ton qui caractérise aussi bien sa manière que sa matière. C'est d'ailleurs un mot de trop qui, pour une bonne partie, vaudra à Viure sa perte.

Jean-Louis Gagnon donne ce ton à une équipe de collaborateurs au verbe haut: Philippe Vaillancourt, Jean-Charles Harvey, Claude-Henri Grignon (alias Valdombre), Pierre Chaloult, Gérard Dagenais et Fernand Lacroix de Montréal ${ }^{2}$. Dans ses mémoires, Gagnon présentera l'initiative comme celle de fils " de bourgeois appauvris par la crise, formés par des clercs, agités de bons sentiments et pressés d'agir »; il ajoutera : "nous cherchions le changement là où il se trouve rarement : à droite ». On est conscient de loger à une autre enseigne que celle $\mathrm{du}$ « nationalisme pur et dur » des Jeune-Canada ou des " états d'âme » de Robert Charbonneau de La Relève, qui venait de paraître en mars et qui avait " pris le temps de réfléchir avant de publier le premier numéro d'une revue dont 
l'influence allait être d'autant plus déterminante, que l'équipe, dans l'ensemble, savait écrire et qu'elle témoignait d'un courage intellectuel certain " par son néocatholicisme ${ }^{3}$.

\section{La rage de vivre contre la peur de vivre}

Le programme non modeste de cette modeste revue est dans son titre qui se met à l'enseigne de Léon Daudet : «Vivre, c'est lutter; qui n'a pas lutté n'a pas vécu». La « juste conception de la vie " est non bourgeoise, désolidarisée du pouvoir de l'argent. La direction de la revue le précise : «En un mot, nous nous proposons de "vivre", et pour vivre, il faut une vie intellectuelle plus intense, il faut renaître. ". Pour ce faire, on annonce une posture anticonformiste: «Vivre, c'est évoluer, c'est-à-dire se défaire de tout esprit conformiste. [...] Nous voulons la révolution des castes intellectuelles. 5 ." Une analogie avec le capitalisme suggère que la jeunesse a été négligée au Canada français : "On a vidé son cerveau et sa personnalité et on lui a bourré le crâne d'idées fabriquées en série. Au lieu de lui enseigner que le capital ancestral devait être doublé, on lui a dit qu'elle devait le laisser moisir en banque. On n'a pas voulu développer en elle le goût de la lutte et la joie de vivre. On a limité sa pensée, on lui a donné un tempérament d'enfant de chœur. » Piaffant d'être dans le vivant et le présent, on évalue que le temps du refoulement est terminé : "Il semble que la vie refoulée graduellement, dans tous les domaines, par les générations qui ont précédé la nôtre, soit arrivée à ce point critique où l'homme ne peut la comprimer davantage "; il n'est plus question d'accepter « les discours menteurs des métèques et des nôtres qui nous félicitent d'être restés un peuple profondément catholique ", ni d'accepter un catholicisme "mécanique ", " un catholicisme de robot». Le mot est lâché : "Vivre, c'est un cri instinctif?." 
Fini le culte des morts à la Barrès, culte qui, consolidant la mémoire du passé, inspire le sens de la nation et de la tradition : «En '35 la jeunesse se divise en deux classes. La première s'étiole à mener une vie petite, bornée par des horizons au ras de la terre et se tourneboule les esprits à chercher des dieux morts. L'autre offre la superficie totale de son épiderme à la vie et demande une destinée. Cette destinée, la jeunesse la veut à tout prix ${ }^{8}$. „ Ces jeunes sentent combien la vie est devenue étroite: "On n'a même pas su nous faire aimer la civilisation; on nous a montré à détester la vie et ce qu'elle apporte de bon, de réconfortant ! En un mot : la béatification du lard salé et des "beans" canayennes a tenu lieu d'intellectualité chez nous pendant tout le siècle dernier'." Ce rapport oppositionnel aux "dieux morts » introduit une idée nouvelle, la vie, le vivant, le présent, idée qui fait son chemin dans la décennie et qui prendra une place centrale dans l'après-guerre.

Vivre, c'est d'abord « combattre la peur de vivre ${ }^{10} »$, c'est dénoncer la grande pesanteur de la peur : « les fils de coureurs de bois se sont mués en froussards, en mioches à qui on fait peur en leur parlant des communistes et du big bad wolf». La peur, c'est " [] grand bobo ${ }^{11}$ ». Face à cette peur, il faut une vie intellectuelle et "afficher une pensée canadienne-française », c'est-à-dire « une pensée française » pour « lutter contre l'ambiance anglaise et surtout américaine ${ }^{12} »$. Dans l'esprit d'Asselin dont L'Ordre est un modèle de retenue et de rigueur, on entend "rester des civilisés : rester Français ${ }^{13}$ » en se payant « de larges rigolades à la Rabelais avec des pointes de scepticisme à la Montaigne » et, à l'occasion quelques tournures d'argot parisien ${ }^{14}$. C'est là un moyen de contrer le "GRAND MIRAGE » du " peuple barbare » que sont les Yankees ${ }^{15}$. C'est aussi une façon de conquérir ce « fameux individualisme, qui ne sait s'affirmer chez nous ni dans les lettres, 
ni dans les arts, ni dans les sciences, c'est-à-dire dans aucun domaine de la pensée ", mais " qui s'exprime bêtement et sans cesse dans le domaine de l'action: en somme exactement le contraire de ce qui devrait se produire ${ }^{16} 》$.

Le projet de « retourner aux sources méditerranéennes et latines dont nous sommes sortis » est la rationalisation pour passer de la France à l'Italie et justifier l'admiration de l'équipe pour Mussolini. L'éclectisme dans la reconnaissance des mấtres - «pas des fossiles " - rappelle l'anxiété avec laquelle on cherche une problématique troisième voie à la Crise et fait imaginer une géopolitique inédite : "Paris est en rectiligne avec Athènes et Rome ; Périclès a un héritier dans Maurras, César dans Lyautey ${ }^{17} »$. Les maîtres étrangers, ce sont Maurras, Lyautey qui donne un empire à la France de Maurras, leur " cher Péguy », Mussolini, Lénine, Hitler, Mustapha Kémal en Turquie. Ce qu'ils aiment au Canada français, c'est «la doctrine de Groulx, les sourires d'Asselin et les gestes larges et pleins de La Vergne ${ }^{18}$ ». Armand La Vergne est pour l'équipe de la revue, tout comme pour les Jeune-Canada, le symbole de la résistance ; il écrit à Gagnon : "Voilà ce que j’ai été, Jean-Louis, rien qu'un humble clairon, sonnant au drapeau quand les Canadiens se relâchaient ou voulaient abandonner la mission sacrée que Dieu a confiée à chacun de nous sur ce continent américain ${ }^{19}$.»

L'abbé Groulx auquel on fait aussi appel consulte René Chaloult avant de cautionner le mouvement; celui-ci écrit à l'abbé : « Leurs idées, parfois personnelles, sont généralement empruntées à Maurras, Daudet et, chez nous, à Asselin. Jeunes et insuffisamment pourvus de sens critique, ils se laissent facilement séduire par toutes les doctrines de leurs idoles. Vaguement partisans d'une laïcisation générale, ils sont de L'Ordre sans réserves. Au reste je les crois catholiques sincères et pratiquants. Comme Asselin qu'ils visitent, ils admirent 
vivement votre carrière d'historien et d'éveilleur national. Je crois toutefois que votre rôle d'apôtre catholique ne les frappe guère $^{20}$. " Groulx, qui est à l'affût des aspirations de tous les mouvements, de l'Association catholique de la jeunesse canadienne-française (ACJC) à La Relève en passant par les JeuneCanada et L'Action nationale, écrit une lettre publique à Gagnon dans laquelle il se dit admiratif de leur "foi dans la vie ", de leur «foi en nos ressources d'âmes » et en leur « catholicisme surtout $^{21} »$. Dans ses Mémoires, il écrira, rétrospectivement : "Je me montrai encourageant pour Vivre au-delà de ce qu'il convenait. Un mot du cardinal Villeneuve me rassura ; j'avais fait les réserves opportunes ${ }^{22}$. 》

Quant à Bourassa, pour Fernand Lacroix, il meurt symboliquement après sa conférence - « Le nationalisme est-il un péché ${ }^{23}$ " - contre les Jeune-Canada : "Henri Bourassa est bien mort, piteusement, dans le champ de l'impérialisme et de l'internationalisme soit-disant [sic] "catholique" hors duquel il n'y a point de salut, étrangler [sic] par un collier de fausses perles. Il reste un conférencier qui tente de nous expliquer son caractère, la main sur le cœur ${ }^{24}$. »

\section{Opposer le corporatisme et le fascisme à la démocratie}

Ces jeunes qui vivent la fin du long régime des libéraux, au pouvoir depuis 1897 , et de celui d'Alexandre Taschereau, en poste depuis 1920, ont déchanté de la démocratie parlementaire, "mystique périmée ", lieu de "combines politiques pour les beaufrèrocraties » et les barons de la finance ${ }^{25}$. L'institution est pour eux « sous la botte du capitalisme des cartels », des "régimes de factions ». Puisque toute "l'histoire de la démocratie se résume dans ce mot : DÉSORDRE», il n'y a qu'un ordre à lui substituer: "l'état corporatiste sous direction autocratique ${ }^{26} \gg$. 
Même si la rédaction affirme que "Vivre est une des premières publications du pays qui se permet d'approuver la dictature hitlérienne ${ }^{27} »$, la revue emprunte à la figure de Mussolini et à l'expérience fasciste italienne le modèle de sa politique $^{28}$. L'organisation politique passe par la structure de l'État qui peut étatiser, par la nation, par le parti et par le chef. À cette vision corporative, on greffe un prosoviétisme circonstancié à opposer au néocapitalisme étatsunien. Dès lors, l'État n'a pas besoin "d'hommes non-producteurs", mais c'est à la nation qu'appartiennent «les créations qui ne sont pas d'une nécessité vitale pour la collectivité ». Dans cet esprit, «[t]out comme il ne s'occupera pas des intellectuels, le parti national-socialiste laissera dans leur paix, les vieillards ». La création d'un parti national-socialiste n'a qu'un but: passer par Mussolini et Lénine pour revenir au « Politique d'abord» de Maurras. Ici, «l'homme appuie son droit sur sa force et non sa force sur son droit $^{29} »$.

Dans ce milieu fascisant, les Juifs sont présentés comme d'éternels "parasite[s] » et c'est le lexique de la pathologie bacille, tuberculose, cancer, peste - qui décrit ceux qui « sont impuissants à s'assimiler à une vie nationale quelconque » et porteurs d'une "forte dose de matérialisme qui désenchante ${ }^{30} \%$.

\section{La vie publique locale}

Le regard de Vivre sur la politique locale est conséquent. Une entrevue imaginaire des Jeune-Canada permet à Fernand Lacroix d'avancer qu'à la hauteur où ils se situent, les JeuneCanada ont peu de chance de rejoindre les masses, thème bien fasciste. L'intervieweur réel observe que le groupe n'a pas de position sur la démocratie et que le nationalisme de Laurendeau est canadien-français et non français. Dans le scénario d'une " sécession ", Lacroix suggère que le corporatisme 
pourrait être l'option politique ${ }^{31}$. J.-L. Gagnon estime quant à lui que leur pensée économique est «faible et navigue dans les incertitudes ». Le Jeune-Canada, qui agit « à coups de dîners-causeries », croit «à l'évolution » alors qu'à Vivre, on croit "à la révolution ». Gagnon soutient qu'il n'y a qu'un seul moyen pour redresser les choses, «la force», et que le Canada français " crève d'un manque de violence ». Il préconise un soulèvement populaire et réalise que c'est «ici qu'arrive l'obligation pour tout révolutionnaire de se barbouiller en démagogue ». Pensant aux Jeune-Canada, il se dit « triste de voir qu'à vingt ans on ne comprenne pas encore que des mains ça se lavent [sic] »; selon lui, les Jeune-Canada retourneront d'où ils sont sortis : «d'un collège de Jésuites. Et l'ACJC comptera un cercle de plus ${ }^{32} »$.

La petite équipe de Vivre ne croit guère en Paul Gouin, chef de la nouvelle Action libérale nationale. Il appartient à "une lignée de joueurs de golf", à la haute bourgeoisie de vieux politiciens, incapables d'une « autre politique, radicale ». Vivre entend par " réformes radicales » le renvoi de toutes les fonctionnaires femmes et leur remplacement par des hommes ; un examen d'entrée obligatoire pour tous les nouveaux fonctionnaires; le nettoyage en règle de tous les services; une limite du nombre de ministères à quatre et des comtés à quarante; des coupures drastiques de salaires; l'annulation du suffrage et son remplacement par le système électif italien et la concentration du pouvoir dans le premier ministre, véritable chef $^{33}$.

Cette prise de position est énoncée dans le dernier numéro de Vivre. Depuis un moment, on avait la petite publication à l'œil. Le cardinal Villeneuve fait publier dans La semaine religieuse un avis à l'effet que la revue «ne respirait pas l'esprit chrétien », ce dont se réjouit Pierre Chaloult, qui conclut que "Vivre respirait l'esprit tout court». 
Mais le mot de trop vint à l'occasion d'un projet de revue à Québec, Ferveur, qu'auraient eu l'intention de lancer Jean-Charles Bonenfant, Bruno Lafleur, Lorenzo Paré, identifiés à L'événement, et Luc Lacoursière. Le périodique n'existait pas encore que Gagnon présentait déjà les futurs rédacteurs comme "dilettantes ", " pédérastes de l'esprit », " chemises roses ", « fifis » et «fausses couches ». L'affaire se retrouva en cour et L'événement du 16 février 1935 titrait «Procès humoristique et littéraire au Palais ». Le juge de première instance estima que la cause pouvait aller au criminel. La famille Chaloult ne pouvait se permettre pour un de ses membres un procès qui finit par ne concerner que Gagnon et qu'il perdit $^{34}$. Le procès n'aida pas au rétablissement financier de Vivre qui disparut avec le numéro du 15 mai non sans avoir annoncé : «Dès septembre prochain nous reprendrons la campagne ».

\section{Les «Cahiers noirs » et Jean-Charles Harvey, auteur des Demi-civilisés}

En septembre 1935, le projet de Gagnon des Cabiers noirs fait aussi long feu. Des cinq numéros annoncés - « Révolution » de J.-L. Gagnon, « Latinité » de Paul Bouchard, « Les responsables" de F. Lacroix, "Les intouchables" de P. Chaloult -, seul parut Jeunesse de Jean-Charles Harvey, préfacé par Gagnon. L'auteur et le cahier ont toutefois du panache.

Harvey, alors âgé de 43 ans, a travaillé à La Patrie et à La Presse de Montréal en 1915 et en 1916 et est entré au Soleil de Québec en 1922, en devenant le rédacteur en chef en 1927. Dans une brochure de 1921, il avait écrit ce qui explique sa relation naturelle à Gagnon et à l'équipe de Vivre: «Exister n'est pas tout : il faut vivre, vivre avec éclat, avec force, avec prestige, avec autorité ${ }^{35}$. » Déjà en 1922, il s'était intéressé à 
la description des mœurs de la ville de Québec dans une chronique du Soleil tenue entre le 14 octobre et le 24 décembre. Il n'est pas toujours facile pour ce caractère intempestif d'être au Soleil, journal du Parti libéral provincial et de Taschereau. Malgré les soupapes qu'il se ménage, Harvey confie à un autre « marginal », son ami le poète Alfred DesRochers : «Il ne nous est plus permis, il me semble, de vivre en marge de la civilisation. Il faut que quelqu'un se lève enfin pour enrayer, pendant qu'il est temps encore, la complète crétinisation d'une race. La tâche en vaut la peine ${ }^{36}$. » Le caractère impersonnel des Canadiens français et des écrivains lui semble être le lieu où le bât blesse : «Dans la plupart des écrits de chez nous, les idées (ou ce qu'on appelle idées) et les sentiments exprimés ne représentent pas la personnalité de celui qui écrit, mais bien les artifices, les préjugés, l'hypocrisie et le psittachisme [sic] de son milieu ${ }^{37}$. »

C'est le monde de la bourgeoisie tant visée par la jeunesse des années 1930 que décrit Harvey dans Les demi-civilisés qui parait le 6 avril 1934 aux Éditions du Totem, dirigées par Albert Pelletier qui publie aussi la revue Les Idées à laquelle Harvey collabore épisodiquement. La trame de ce roman de la ville gravite autour de la fondation par Max Hubert d'une revue au titre évocateur, Vingtième siècle, présentée comme une "entreprise de libération »: "Pour la première fois, en ce pays de l'impersonnel et de l'artifice, où seule la pensée officielle avait eu droit de cité, paraissait une publication vraiment libre, ouverte à toutes les opinions sensées, rompant le conformisme accepté, depuis un siècle et demi, par le troupeau servile ou terrifiée ${ }^{38}$. » Le défi de l'affirmation de l'individu est au centre du projet: «Plus nettement que jamais, je me rendis compte que j'étais un rebelle. Pourquoi rebelle? Parce que je refusais d'abdiquer le moi, ce moi qui prenait des proportions infinies à mesure que je comptais, méprisant ou 
apitoyé, les infirmités et les insignifiances du monde qui m'entourait ${ }^{39}$. » Le personnage principal du roman a su être capable de doute: "On vous a toujours dit: "Ne doutez pas !" Moi, je vous dis : "Doutez !" C'est la planche de salut de l'intelligence, c'est la ligne de flottaison de l'être raisonnable. Créez en vous la belle et courageuse inquiétude qui vous épargnera la maladie du sommeil et vous conduira à des trouvailles splendides ${ }^{40}$. » C'est aussi « un signe de force spirituelle que de ne pas se laisser posséder par l'esprit de corps ou le fanatisme de la famille». Et Max Hubert d'ajouter: «Pour juger la valeur d'une nation, il faut se placer solidement sur le plan humain $^{41}$.»

Deux semaines plus tard, le roman est condamné par le cardinal Villeneuve de Québec, puis dans d'autres diocèses, sauf celui de Montréal. Harvey est immédiatement expulsé du Soleil. Pour le dédommager, Taschereau lui propose la direction de la bibliothèque de l'Assemblée législative. L'archevêché s'oppose à cette nomination à une institution intellectuelle. On permute alors le colonel Marquis du Bureau de la Statistique à la bibliothèque et Harvey s'en va au Bureau de la Statistique. Confirmation d'un monde de demi-civilisés. Avec l'arrivée au pouvoir de Maurice Duplessis, Harvey perdra son emploi, quittera Québec pour aller fonder Le Jour en $1937^{42}$.

C'est donc cet homme, qui a dénoncé l'étroitesse d'esprit, critiqué le clergé et promu tout autant la libre pensée qu'une certaine sensualité, qui publie Jeunesse dans les «Cahiers noirs » des Éditions de Vivre ${ }^{43}$. Gagnon, qui signe l'introduction, prend le contre-pied de ce que Maritain avait proposé à certains jeunes de Québec, au restaurant Kerhulu : «Commencez par exister. Il faut être d'abord ${ }^{44}$. » Gagnon affirme : «Nous devons vivre - exister est un état de fossile» et dit son intérêt pour la pensée de Harvey: «Ce que nous 
aimons surtout chez Harvey, c'est qu'il ne s'embarrasse pas de faux dogmes et de dieux morts. Au lieu de se contenter des plaisirs conformistes du fonctionnarisme, il demande sa part à la vie - la part du risque et de la guerre ${ }^{45}$. » Avant de conclure, il donne au lecteur un indice de l'origine du qualificatif des «Cahiers »: "J'ai vu quelque chose de noir - une jeunesse qui s'écœure. Je ne propose aucun remède. Je ne m'habille pas d'une chemise noire, pas plus que je ne porte en sautoir un marteau agrémenté d'une faucille; je ne prépare pas un discours pour 1937, et je ne me suis jamais baladé dans les alentours de Downing Street. Cependant je suis convaincu qu'il faut faire quelque chose. La jeunesse a bien du $\mathrm{mal}^{46} . »$

La première mouture - brève - de la conférence est d'un ton plus radical ; Harvey, qui vient de subir les foudres cléricales, dénonce l'éducation qui a produit « une multitude de désaxés » et « de désœuvrés » et souligne combien la Crise a créé « tant d'impatience chez les jeunes». Pour lui, les classes dirigeantes n'ont rien fait d'autre que «de préparer les cœurs à l'émeute et les bras à la révolution »; il «faut nécessairement que l'explosion se produise ${ }^{47} \%$.

Dans le texte du "Cahier noir », Harvey estime que les jeunes ont été oubliés, condamnés qu'ils sont depuis leur sortie des collèges à être des " chasseurs de place ». Il se dit convaincu que «tout est pourri dans ce monde et qu'il n'existe rien de sain, de généreux, de désintéressé parmi les chefs qui ont eu le bonheur ou le malheur de naitre quinze ou vingt ans avant » la présente génération. À la crise politique s'est ajoutée une crise économique; les jeunes "se sont demandé si notre régime économique qui les affamait ou les accrochait aux basques de leurs parents, était bien adapté aux conditions actuelles ». Harvey continue à trouver le système capitaliste «le moins imparfait ». S'il voit d'un oil différent ce qui s'est 
passé en Allemagne, il reconnaît que la jeunesse y est pour quelque chose : "Il est vrai qu'on ne peut comparer la jeunesse du nouveau monde, surtout celle du Canada français, à celle des vieux pays, plus tourmentés, plus habituellement tiraillés par les doctrinaires, mais je n'oublie pas que les graves événements de l'Allemagne qui ont porté Hitler au pouvoir avec sa dictature et qui, aujourd'hui, menacent la paix du monde, sont dûs [sic] principalement à la coalition de toutes les jeunesses ». Il dit craindre aussi ce qui se passe de ce côté de l'Atlantique : «Ce qui se prépare dans nos agglomérations urbaines, ce n'est pas seulement la haine contre un état de choses ou contre des lọis, mais aussi la rupture entre deux générations. Maintes expressions d'opinions me font croire que les jeunes en veulent à leurs devanciers d'occuper seuls les emplois ${ }^{48}$. »

Ce premier «Cahier noir » ne laisse pas les gens de La Relève indifférents. Robert Charbonneau estime que "cette œuvre n'est pas le résultat d'un vrai contact avec la jeunesse ». Au contraire de Harvey, il pense que « [1]a jeunesse n'est pas toute anticapitaliste». Ce dernier "semble attribuer la crise actuelle à la fatalité et c'est en quoi il s'oppose aux jeunes qui croient que le libéralisme économique, qui a été le moteur de notre capitalisme, et sa doctrine, n'est qu'un aspect d'une crise dans l'homme, qui date de la renaissance. M. Harvey laisse entendre que si les jeunes s'attaquent au capitalisme, c'est qu'il est devenu incapable de leur prodiguer le luxe et les satisfactions matérielles »; il devrait savoir « que le capitalisme pour un Daniel-Rops, un Maritain, un Mounier importe moins que le retour aux valeurs spirituelles de charité et de bonté ${ }^{49}$ ».

Dans l'introduction à Jeunesse, Gagnon écrivait : « Pour des raisons qu'il est plus difficile de deviner que de dire, nous sommes obligés de couper court à notre publication bi-mensuelle. Cependant, il se pourrait - si les dieux ne nous sont 
pas défavorables - que Vivre refasse un bond et retombe sous forme d'hebdomadaire ${ }^{50}$. " Le rebond allait s'appeler $\mathrm{La} \mathrm{Na}$ tion.

\section{$* * *$}

Il n'est pas facile de conclure à propos de Vivre, périodique mensuel, éclectique et de courte durée dont les positions seront en partie reprises par l'hebdomadaire La Nation. L'éclectisme des sources d'inspiration de la revue est d'abord un signe de la crise non plus seulement financière, économique et sociale des années 1930, mais tout autant de la crise politique et spirituelle ou intellectuelle qui traverse la décennie. Au-delà de la personnalité même de son rédacteur principal, Jean-Louis Gagnon, et de la facilité relative avec laquelle, selon René Chaloult, ses rédacteurs se laissent séduire par les courants d'idées européens, la revue cherche anxieusement un nouvel ordre après avoir critiqué le désordre induit par le capitalisme «bourgeois » et par le communisme. La voie de sortie de la crise est loin d'être évidente pour les contemporains et les emprunts éclectiques au corporatisme, au fascisme, au soviétisme montrent bien comment l'architecture et les matériaux de la reconstruction ne peuvent être qu'improvisés pour ces « révolutionnaires » qui se démarquent eux-mêmes de La Relève et des Jeune-Canada, reconnaissant du coup l'émergence d'une nouvelle constellation de mouvements de jeunes.

La religion n'est un objet de préoccupation ni pour les Jeune-Canada ni pour ces francs-tireurs, pas suffisamment religieux aux yeux de Chaloult et de l'abbé Groulx qui n'a pas, publiquement, de mots décourageants ou négatifs à leur égard. La jeunesse de l'époque préoccupe un aîné, Jean-Charles Harvey, qui publie le seul «Cahier noir» des éditions de Vivre. La jeunesse dont Harvey attend beaucoup n'a pas à voir comme 
tel avec les mouvements d'action catholique qui émergent alors, la Jeunesse ouvrière catholique en 1931, la Jeunesse étudiante catholique en $1935^{51}$; mais manifestement, il y a chez Harvey et chez les rédacteurs de Viure une conscience d'appartenance à une génération, à une relève, à une jeunesse canadienne-française.

Vivre se démarque de façon provocatrice des «états d'âmes " des jeunes catholiques de La Relève qui, leur reconnait-on, ont l'avantage de la plume. Un des directeurs de $\mathrm{La}$ Relève, prend la peine de relever le gant et d'expliquer que la crise n'est pas qu'économique ou matérielle. Reprenant la réflexion sur l'humanisme intégral de Jacques Maritain, réflexion dont le philosophe a offert la primeur à une certaine jeunesse de Montréal, à l'automne 1934, Robert Charbonneau rappelle que la crise est plus profonde, qu'elle est spirituelle et que l'homme « intégral » était celui du Moyen-Âge, à l'esprit duquel il faut revenir.

Le «nationalisme pur et dur» des Jeune-Canada est passé au crible de l'ironie des rédacteurs de Vivre. Pour la revue, les allures bourgeoises des Jeune-Canada font de ceuxci un cercle particulier de l'ACJC et rien de plus révolutionnaire. Mais en quoi Vivre est-elle "révolutionnaire" ?

La revue a d'abord un ton et un lexique, ceux de nonconformistes exaspérés par le pourrissement de la situation. La dénonciation des "joueurs de golf», des "chemises roses » ou des "pédérastes de l'esprit » rend compte de leur fin de non-recevoir de la tradition.

Jean-Louis Gagnon est le premier à identifier la mentalité de "peur» des Canadiens français, à proposer que vivre veut dire « combattre la peur » qui est le " grand bobo », l'empêchement fondamental de ses concitoyens. Il inaugure en cela une tradition que reprendront Paul Bouchard, Dostaler O'Leary, Paul-Émile Borduas, autant de figures d'une criti- 
que assez radicale et d'une prise de position politique indépendantiste, dans les deux premiers cas.

Vivre, c'est encore le plaidoyer pour la vie, pour le vivant. Vivre est nécessairement une réalité du présent et, du coup, une dénonciation obligée de la vie refoulée, du passé, du culte des morts, des fossiles. Leur maître est de moins en moins le passé, de plus en plus le présent.

La radicalité de Vivre réside dans sa découverte de l'État au terme d'une critique de la démocratie parlementaire, du capitalisme comme du communisme ou du socialisme d'État et au terme de l'adhésion au corporatisme dont la régulation se fait par l'État. Du coup, l'État devient le levier politique dont ont besoin ces séparatistes. C'est leur façon de revenir, malgré des détours fort éclectiques, au "Politique d'abord" de Maurras. Il faut comprendre qu'en 1935, un positionnement indépendantiste ne peut pas ne pas faire découvrir l'État dont un capitalisme et un vieil ultramontanisme ambiants bloquaient la découverte et la valorisation. Vivre offre un cas peu connu et peu fréquent de corporatisme politique dans un Canada français où le corporatisme inspiré de Quadragesimo Anno (1931) puis d'expériences nationales variées (Italie, Portugal, Espagne) est d'abord et avant tout socia ${ }^{2}$.

Pour tenir tant de positions inédites, il fallait d'abord que ces rédacteurs et que leur mentor, Jean-Charles Harvey, soient des individus affirmés tout autant dans leur pensée que dans leur action. Un individu s'esquissait au temps même de Saint-Denys Garneau, un sujet paraissait devoir être souverain, d'abord individuellement avant de pouvoir penser l'être collectivement. 


\section{NOTES}

${ }^{1}$ André-J. Bélanger consacre quelques pages intéressantes à Vivre dans L'apolitisme des idéologies québécoises. Le grand tournant de 1934-1936, Québec, Les Presses de l'Université Laval, 1974, p. 329-335.

${ }^{2}$ Hector de Saint-Denys Garneau, poète en formation qui publiera Regards et jeux dans l'espace en 1937 et qui collabore à La Relève avec ses collègues du Collège Sainte-Marie, n'écrit pas dans Vivre qui bénéficie par ailleurs de ses talents de peintre. Garneau fournit la maquette de couverture de la revue en octobre puis en novembre 1934. Voir Giselle Huot, «L'aventure artistique du peintre de Saint-Denys Garneau ", Mens : Revue d'bistoire intellectuelle de l'Amérique française, vol. IV, $\mathrm{n}^{\circ} 2$ (printemps 2004), p. 252-253.

${ }^{3}$ Jean-Louis Gagnon, Les apostasies. I : Les coqs de village, Montréal, La Presse, 1985, p. 66-68 ; Gagnon qui a fait ses études classiques aux collèges SainteMarie et Brébeuf de Montréal vient d'obtenir son baccalauréat (1935) de l'Université d'Ottawa ; comme en témoignent ses mémoires, son parcours intellectuel et politique fut placé à l'enseigne de la sinuosité. À l'annonce de la disparition de la revue, Gagnon en fait l'histoire : «Vivre », Vivre, vol. II, n 5 (15 mai 1935). Vivre, pressée de se mesurer avec la vie, laissait à La Relève « le combat avec l'ange "; à la première revue, on « était déjà des journalistes, pour le meilleur ou pour le pire, alors qu’à $L a$ Relève, on faisaìt déjà des livres ", JeanLouis Gagnon, «En ce temps-là... », Études françaises, vol. V, n 4 (novembre 1969), p. 462.

${ }^{4}$ La Direction, «Vivre », Vivre, vol. I, n" 1 (mai 1934).

${ }^{5}$ La Direction, «Vivre », Vivre, vol. II, n” 2 (22 mars 1935).

"Jean-Louis Gagnon, "Des maîtres pas des fossiles ", Vivre, vol. I, n" 3 (octobre 1934).

${ }^{7}$ «Vivre», position reproduite dans L'Ordre, 11 octobre 1934.

${ }^{8}$ La Direction, «Vivre », Vivre, vol. II, n"1 (8 mars 1935).

${ }^{9}$ La Direction, « Vivre », Vivre, vol. II, n” 2 (22 mars 1935).

"Jean-Louis Gagnon, « Vivre », Vivre, vol. I, n" 1 (novembre 1934).

${ }^{11}$ Gagnon, « Des maîtres pas des fossiles ».

${ }^{12}$ La Direction, "Vivre », Vivre, vol. I, n" 1 (mai 1934) ; leur France n'est pas celle qui a été " pieusement » décrite par le conservatisme des clercs et de 
nombreux politiciens et journalistes, La Direction, « Vivre », Vivre, vol. II, $\mathrm{n}^{\text {" }}$ 2 (22 mars 1935).

${ }^{13}$ La Direction, « Vivre », Vivre, vol. I, n” 4 (novembre 1934).

${ }^{14}$ Jean-Louis Gagnon, « Folies françaises, I », Vivre, vol. I, n 5 (décembre 1934).

${ }^{15}$ Claude Berre Jean-Louis Gagnon], «Vive l'Amérique ! », Vivre, vol. I, n” 1 (mai 1934).

${ }^{16}$ La Direction, «Vivre », Vivre, vol. I, n” 7 (février 1935).

${ }^{17}$ Gagnon, «Folies françaises, I ».

${ }^{18}$ La Direction, «Vivre », Vivre, vol. I, n" 5 (décembre 1934) ; Rex Desmarchais, "Olivar Asselin. Le sens d'un sourire », Vivre, vol. I, n" 4 (novembre 1934).

19) Armand La Vergne, « Propos d'un jeune... un peu mûr », Vivre, vol. I, n" 6 (janvier 1935); et avec un ton nietzschéen, Jean-Louis Gagnon, « Ainsi parlait La Vergne ", Vivre, vol. II, n 2 (22 mars 1935).

${ }^{20}$ Lettre de René Chaloult à l'abbé Lionel Groulx, 12 octobre 1934 reproduite dans Lionel Groulx, Mes mémoires, Montréal, Fides, 1972, III, p. 290. Jeune avocat et futur député de Kamouraska (1936-1939), René Chaloult fut une des rares têtes de pont de la jeunesse nationaliste à Québec, exposée à la partisannerie de " la clique des Taschereau » et à ses aléas ainsi qu'au milieu indifférent des étudiants universitaires que l'abbé Camille Roy a à l'œil. Chaloult collabore, à ses risques, à L'Action française de Montréal à compter de 1923. Voir à ce sujet Groulx, Mes mémoires, II, p. 137-146.

${ }^{21}$ Abbé Lionel Groulx au directeur, "Vivre ", L'Action nationale, vol. III, n" 11 (novembre 1934), p. 171-176 ; Vivre, vol. I, n" 4 (novembre 1934), reproduite dans Gagnon. Les apostasies, p. 258-260.

${ }^{22}$ Groulx, Mes mémoires, III, p. 291.

${ }^{23}$ Henri Bourassa, «Le nationalisme est-il un péché ?», Le Devoir, $1^{\text {er }}$ mai 1935 ; «Le nationalisme dans l'Église », Le Devoir, 10 mai 1935 ; « Catholiques et non-catholiques », Le Devoir, 16 mai 1935.

${ }^{24}$ Fernand Lacroix, « Bourassa est mort », Vivre, vol. II, n” 5 (15 mai 1935).

${ }^{25}$ La Direction, « Vivre », Vivre, vol. I, n" 4 (novembre 1934).

${ }^{26}$ Jean-Louis Gagnon, «Les beautés du régime », Vivre, vol. I, n" 5 (décembre 1934). 
${ }^{27} \mathrm{La}$ Direction, « Pour la réconciliation de la vérité », Vivre, vol. I, n 5 (décembre 1934).

${ }^{28}$ On préfère Mussolini, qui s'est fait de ses propres forces, à Hitler, porté par Goering et Goebbels : Pierre Chaloult, « Sous le signe de la Swastika », Vivre, vol. I, n 3 (octobre 1934).

${ }^{29}$ Jean-Louis Gagnon, « Politique », Vivre, vol. II, n” 3 (12 avril 1935).

${ }^{30}$ Pierre Chaloult, « Israël », Vivre, vol. II, no 3 (12 avril 1935).

${ }^{31}$ Fernand Lacroix, «Le Jeune-Canada parle », Vivre, vol. II, n" 4 (26 avril 1934).

${ }^{32}$ Jean-Louis Gagnon, « Politique : les Jeune-Canada », Vivre, vol. II, n" 4 (26 avril 1935) ; La Direction, «Vivre », Vivre, vol. II, n” 4 (26 avril 1935).

${ }^{33}$ La Direction, « Paul Gouin êtes-vous sérieux ? », Vivre, vol. II, n” 5 (15 mai 1935).

${ }^{34}$ Gagnon décrit les péripéties de ce procès dans ses mémoires, Les apostasies, p. 101-105; « Messieurs, voici le courrier », Vivre, vol. I, n 7 (février 1935); Jean-Louis Gagnon, «Bravo, bravissimo, bravisibisbruno », Vivne, vol. II, $\mathrm{n}^{\circ}$ 1 (8 mars 1935) ; du même, "Nous avons fait un beau libelle », Vivre, vol. II, n"1 18 mars 1935); Cour des Sessions de la Paix, district de Québec, [juge Laetare Roy], « Enquête préliminaire », Division des archives de l'Université Laval, fonds Jean-Louis Gagnon, P426/B1/1, dossier 312 ; Lucien Parizeau, «L'affaire de Vivre», L'Ordre, 5 mars 1935.

${ }^{35}$ Dans La chasse aux millions : l'avenir industriel du Canada français, cité dans Jean-Charles Harvey, Les demi-civilisés, édition critique par Guildo Rousseau, Montréal, Presses de l'Université de Montréal (Bibliothèque du Nouveau Monde), 1988, p. 18.

${ }^{36}$ Jean-Charles Harvey à Alfred DesRochers, 2 mars 1931, cité dans ibid., p. 27.

${ }^{37}$ Jean-Charles Harvey, « Le courage d'une opinion », Le Soleil, 24 mai 1933, cité dans ibid., p. 16.

${ }^{38} \mathrm{Je}$ cite l'édition de 1962, paru aux Éditions de l'Homme, avec une introduction de Harvey, p. 63.

${ }^{39}$ Ibid., p. 53.

40 Ibid., p. 87.

${ }^{41}$ Ibid., p. 127. 
${ }^{42}$ Sur les péripéties de cette condamnation, Yves Lavertu, "Les demi-civilisés », dans Pierre Hébert, Yves Lever et Kenneth Landry, dir., Dictionnaire de la censure au Québec. Littérature et cinéma, Montréal, Fides, 2006, p. 179-183 ; sur la condamnation du journal L'Ordre d'Olivar Asselin le 4 mai 1935, l'analyse d'Hélène Pelletier-Baillargeon, ibid, p. 505-507. Un des rares journalistes à résister à la mise au silence de la presse, Berthelot Brunet, parlera d'un Québec qui « a brisé les Tables de la Loi ». Berthelot Brunet, «Quand Québec se dessale », L'Ordre, 25 avril 1934.

${ }^{43} \mathrm{Il}$ s'agit à l'origine d'une conférence faite à Québec le 6 juin 1935 dont rend compte Le Soleil du lendemain ; des aperçus paraissent dans Viure du 15 mai 1935 et dans Les Idées de juin 1935.

${ }^{44}$ On se souviendra que c'est Harvey lui-même qui avait rendu compte de cette rencontre : «La métaphysique chrétienne de Jacques Maritain », Le Canada, 16 octobre 1934.

${ }^{45}$ Dans Jean-Charles Harvey, Jeunesse, [Québec], Éditions de Vivre, 1935, p. 8.

${ }^{46}$ Ibid.

${ }^{47}$ Jean-Charles Harvey, « La crise de la jeunesse », Vivre, vol. II, n" 5 (15 mai 1935).

${ }^{48}$ Les passages entre guillemets se trouvent successivement dans Harvey, Jeunesse, p. 20, 22, 25, 30, 26, 21.

49 Robert Charbonneau, "Les cahiers noirs ", La Relève, deuxième série, deuxième cahier (octobre 1935), p. 56-58.

${ }^{50}$ Harvey, Jeunesse, p. 13.

${ }^{51}$ Louise Bienvenue, Quand la jeunesse entre en scène : l'Action catholique avant la Révolution tranquille, Montréal, Boréal, 2003, 291 p.

${ }^{52}$ Pierre Trépanier, " Quel corporatisme (1820-1965) ? ", Les Cabiers des Dix, n 49 (1994), p. $180-183$. 